\title{
PRIMARY VESICOURETERIC REFLUX IN CHILDREN BETWEEN 1 MONTH AND 12 YEARS WITH URINARY TRACT INFECTION ADMITTED IN A TERTIARY CARE HOSPITAL
}

Subbiah. M, Jenitha. B, Prabu Elango P. R, Nagarajan. C.

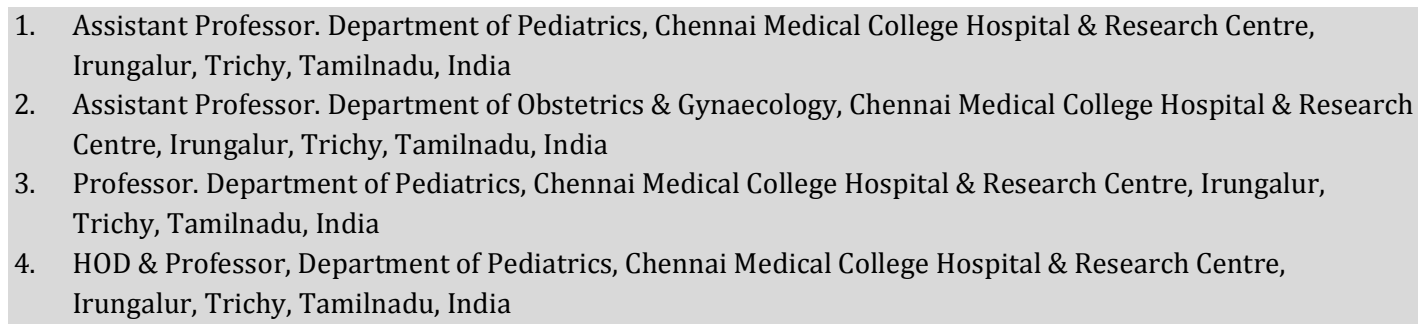

\section{CORRESPONDING AUTHOR:}

Dr. Subbiah. M.

B-5 staff Quarters,

Chennai Medical College Hospital,

Irungalur, Trichy- 621105.

Email: drmsubbiah@yahoo.co.in

ABSTRACT: OBJECTIVE: To determine the proportion of primary vesicoureteric reflux in children between 1 month and 12 years with urinary tract infection admitted in a tertiary care hospital. METHODOLOGY: A Hospital based descriptive cross sectional study was conducted between May 2010 and October 2011 in the Department of pediatrics, Chennai Medical College Hospital, Trichy. Convenient sampling technique was applied to select the study subjects. Renal ultrasound and voiding cystourethrography (VCUG) examinations were performed in all confirmed cases of first UTI under 5 years of age and recurrent UTI at any age. Any children with underlying anatomic abnormalities of urinary tract or neurogenic abnormalities were excluded from the study. The severity of reflux expressed as a grade, which is based on the system established by the International Reflux Study in Children. The data was analyzed by proportions and chi - square test using SPSS statistical package version 11 at $95 \%$ confidence interval. P value $<0.05$ was considered significant. RESULTS: First UTI commonly occurred under 2 years of age (54\%). Recurrent UTI predominantly occurred beyond 2 years of age (70\%).In less than 2 years age group the percentage of UTI was slightly higher in males(53\%) than females(47\%).After 2 years females had a higher percentage(64\%) of UTI than males (36\%).In first UTI under 2 years fever was the presenting complaint in $90 \%$ cases. In recurrent UTI fever was seen only in $28.5 \%$ cases. Common presentations of recurrent UTI under 2 years were irritability (64.3\%), vomiting (42.8\%), poor feeding (35\%) and failure to thrive (35\%).Common presentations of recurrent UTI in older children were specific urinary symptoms such as increased frequency (70\%), dysuria (60\%) and abdominal pain (45\%).In first UTI only $22.5 \%$ cases were presented with VUR whereas in recurrent UTI 32\% cases were presented with VUR. Grading of VUR in our study was Grade I 41\%, Grade II 32\%, Grade III 9\%, Grade IV 9\% and Grade V 9\%. In first UTI severe Grades of VUR ( $>$ Grade 2) were not seen. The sensitivity and specificity of ultrasound in detecting VUR were $36.4 \%$ and $100 \%$ respectively. CONCLUSION: Since the Vesicoureteric Reflux appears to be more common in the recurrent UTI, Voiding cystourethrogram (VCUG) is recommended in all the cases of recurrent UTI, so that prophylaxis can be entertained to prevent renal scarring and chronic glomerular insufficiency. Even in the first UTI, if the child is not 
shown improvement as expected, VCUG can be done. Since USG is safe noninvasive it is recommended in all confirmed cases of UTI.

INTRODUCTION: Urinary tract infection (UTI) is a common bacterial infection in infants and children. The risk of having a UTI before the age of 14 years is approximately $1-3 \%$ in boys and $3-10 \%$ in girls ${ }^{1}$. The diagnosis of UTI is often missed in infants and young children, as urinary symptoms are minimal and often nonspecific. Rapid evaluation and treatment of UTI is important to prevent renal parenchymal damage and renal scarring that can cause hypertension and progressive renal damage ${ }^{2}$. Risk factors for renal scarring include : young age especially children less than one year old, delay in initiating antibacterial treatment, recurrences of UTI, and presence of moderate to severe vesicoureteric reflux [VUR]. Some children, who present with an apparently uncomplicated first UTI, turn out to have a significant reflux. Subclinical infections can sometimes lead to severe bilateral renal scarring. Therefore even a single documented UTI in a child must be taken seriously. It is recommended that all infants with UTI should be screened by ultrasonography, followed by VCUG and DMSA scintigraphy. Children older than 5 years are screened by ultrasonography and further evaluated only if this is abnormal. It is emphasized that patients with recurrent UTI at any age should undergo detailed imaging with ultrasonography, VCUG and DMSA scintigraphy3. Reflux nephropathy occurs more commonly in children with UTI than those with sterile urine. In children with UTIs, the reported frequency of VUR varies from $20 \%$ to $40 \%{ }^{4-7}$.VUR by itself appears to be harmless but in conjunction with UTI it can have devastating effects on the kidneys. However when reflux is diagnosed earlier and managed appropriately renal insufficiency is rare. Hence the study was conducted to study the proportions of cases of VUR in UTI.

METHODS: This was a prospective, descriptive study conducted in Chennai Medical College Hospital \& Research Institute, Trichy between May 2010 and October 2011.All children with suspected UTI were recruited and they were subjected to detailed history and thorough clinical examination. Any child with underlying anatomic abnormalities of urinary tract or neurogenic abnormalities was excluded. The total number of patients was 78. Renal function tests and urine analysis were taken in all children. Urine culture by suprapubic aspiration was done in children under 5 years and midstream urine was collected in children above 5 years. Any growth of bacteria in suprapubic aspiration was considered significant. All cases were subjected to USG abdomen. Renal ultrasound was considered suggestive of vesicoureteric reflux (VUR) if dilatation of pelvi-calysis, dilatation of the ureter or dilatation of collecting system of one or both kidney was reported. All children with positive urine culture were given antibiotics for 14 days. Voiding Cystourethrography (VCUG) was performed after 2 weeks for all children with first UTI under 5 years of age, in children $>5$ years with first UTI if USG abdomen shows abnormalities and in all children with recurrent UTI irrespective of age. VUR was classified as per international VUR classification ${ }^{8}$. Radiologists read all imaging studies. Proportion of outcome measures arrived as percentages. To compare groups chi - square test was used. $\mathrm{P}<0.05$ was considered for statistical significance.

RESULTS: In our study totally 78 children were included. In children with first UTI we found that most of them presented under 2 years (54\%). 46\% in the age group of 3-5 years. None of the children presented after the age of 5 years. In contrast to the first UTI most of the children in the recurrent UTI presented at a later age group ( $42.5 \%$ after 6 years). Among 78 children 34 (43.6\%) 
were males and 44 (56.4\%) were females. Under 2 years the percentage of UTI was slightly higher in males (53\%) than females (47\%).After 2 years females had a higher percentage of UTI than males. In the age group of 3-5 years UTI was observed in $62.5 \%$ of females \& above 6 years $65 \%$ were females.

TABLE 1 Age and Sex distribution of UTI

\begin{tabular}{|l|l|l|l|l|l|l|l|}
\hline \multirow{2}{*}{ Age Group } & \multicolumn{3}{|l|}{ First time UTI } & \multicolumn{3}{l|}{ Recurrent UTI } & Total \\
\cline { 2 - 8 } & Male & Female & Total \% (n=31) & Male & Female & Total \% (n=47) & No \% (n=78) \\
\hline 1 mo-2 yr & 11 & 9 & $\begin{array}{l}20 \\
(66.6 \%)\end{array}$ & 7 & 7 & $\begin{array}{l}14 \\
(29.7 \%)\end{array}$ & $\begin{array}{l}34 \\
(43.5 \%)\end{array}$ \\
\hline $3-5 y r$ & 6 & 5 & $\begin{array}{l}11 \\
(33.4 \%)\end{array}$ & 3 & 10 & $\begin{array}{l}13 \\
(27.6 \%)\end{array}$ & $\begin{array}{l}24 \\
(30.7 \%)\end{array}$ \\
\hline $6-12 \mathrm{yr}$ & 0 & 0 & $\begin{array}{l}0 \\
(0.0 \%)\end{array}$ & 7 & 13 & $\begin{array}{l}20 \\
(42.5 \%)\end{array}$ & $\begin{array}{l}20 \\
(25.6 \%)\end{array}$ \\
\hline Total & 17 & 14 & $\begin{array}{l}31 \\
(100 \%)\end{array}$ & 17 & 30 & $\begin{array}{l}47 \\
(100 \%)\end{array}$ & $\begin{array}{l}78 \\
(100 \%)\end{array}$ \\
\hline
\end{tabular}

$\left(\mathrm{x}^{2}\right.$ value 2.17 at $\left.95 \% \mathrm{CI} \mathrm{Df}=2 \mathrm{p}=0.337\right)$

Fever was the most common symptom in children who presented with first UTI. Out of the 20 children in less than 2 years age group with first UTI, 18 of them (90\%) had fever. Irritability $(50 \%)$, diarrhea $(25 \%)$ and poor feeding $(15 \%)$ were the other common complaints. In 3-5 years age group also still fever was the predominant complaint (54.5\%). Increased frequency $(27.3 \%)$, dysuria (18.2\%), and abdominal pain (18.2\%) were the next common presenting complaints. In recurrent UTI most of the children under 2 years presented with nonspecific symptoms such as irritability (64\%), vomiting (42.8\%) and poor feeding (35.7\%), but the proportion of children with fever was low (28.5\%). In older children ( $>2$ years) with recurrent UTI most of them presented with specific urinary symptoms like increased frequency (77\%), dysuria (70\%), and abdominal pain (38.5\%).

TABLE 2: AGE DISTRIBUTION OF VUR

\begin{tabular}{|l|l|l|l|}
\hline Age & First UTI & Recurrent UTI & Total \\
\hline 1 month - 2 years & $4 / 20(25 \%)$ & $7 / 14(50 \%)$ & $11 / 34(32.3 \%)$ \\
\hline 3-5 years & $3 / 11(27.3 \%)$ & $5 / 13(38.5 \%)$ & $8 / 24(33.3 \%)$ \\
\hline 6-12 years & - & $3 / 20(15 \%)$ & $3 / 20(15 \%)$ \\
\hline Total & $\mathbf{7 / 3 1 ( 2 2 . 5 \% )}$ & $\mathbf{1 5 / 4 7}(\mathbf{3 2 \% )}$ & $\mathbf{2 2 / 7 8 ( 2 8 . 2 \% )}$ \\
\hline
\end{tabular}

Of the 78 cases with UTI, 22 cases were found to have VUR on VCUG. Among first UTI the VUR was seen in $22.5 \%$ cases. In the recurrent UTI, $32 \%$ of cases were found to have VUR on VCUG. The results were not statistically significant $(\mathrm{p}=0.348)$. Among first UTI, 4 cases $(25 \%)$ of VUR were seen in less than 2 years and 3 cases (27.3\%) in the age group of 3-5 yrs. No VUR was seen 
after 5 years. In contrast among recurrent UTI, $50 \%$ of VUR were seen in less than 2 yrs. In the age group of 3-5 \& above 6 years the incidence of VUR was $38.5 \%$ and $15 \%$ respectively.

TABLE 3: SEX DISTRIBUTION OF VUR

\begin{tabular}{|l|l|l|l|}
\hline & Male & Female & Total \\
\hline First UTI & $3 / 15(20 \%)$ & $4 / 16(25 \%)$ & $7 / 31(22.5 \%)$ \\
\hline Rec UTI & $4 / 19(21 \%)$ & $11 / 28(39.2 \%)$ & $15 / 47(32 \%)$ \\
\hline Total & $\mathbf{7 / 3 4}(\mathbf{2 0 . 6 \% )}$ & $\mathbf{1 5 / 4 4}(\mathbf{3 4 \% )}$ & $\mathbf{2 2 / 7 8 ( 2 8 \% )}$ \\
\hline
\end{tabular}

Among the total 22 cases of VUR, 7 cases were males and 15 were females. In the first UTI group there wasn't any significant difference between the males and females (Males 3 cases, females 4 cases), whereas in recurrent UTI females had a higher incidence of VUR compared to males (Females 11, Males 4).

\section{TABLE4: SENSITIVITY AND SPECIFICITY OF USG IN DETECTION OF VUR}

\begin{tabular}{|l|l|l|l|}
\hline \multirow{2}{*}{ USG } & \multicolumn{2}{|l|}{ VCUG } & \multirow{2}{*}{ Total } \\
\cline { 2 - 3 } & Positive & Negative & \\
\hline Positive & 8 & 0 & 8 \\
\hline Negative & 14 & 56 & 70 \\
\hline Total & $\mathbf{2 2}$ & $\mathbf{5 6}$ & $\mathbf{7 8}$ \\
\hline
\end{tabular}

Among 78 children who had undergone USG, 8 children had mild to moderate ureterohydronephrosis and all of them had VUR on VCUG. Among these children in whom USG was able to pick up VUR, 6 of them had significant VUR of Grades 3 and above on VCUG. On the other hand in 14 children with VUR on VCUG, the renal ultrasound was normal. The sensitivity of ultrasound for detection of VUR was $36.4 \%$; specificity was $100 \%$. The positive predictive value of ultrasound for VUR was $100 \%$ and the negative predictive value was $80 \%$.

TABLE 5: GRADING OF VUR

\begin{tabular}{|l|l|l|l|l|l|l|}
\hline & Grade I & II & III & IV & V & Total \\
\hline First UTI & 4 & 3 & - & - & - & 7 \\
\hline Rec UTI & 5 & 4 & 2 & 2 & 2 & 15 \\
\hline Total & $\mathbf{9 ( 4 1 \% )}$ & $\mathbf{7 ( 3 2 \% )}$ & $\mathbf{2 ( 9 \% )}$ & $\mathbf{2 ( 9 \% )}$ & $\mathbf{2 ( 9 \% )}$ & $\mathbf{2 2}$ \\
\hline
\end{tabular}

Among 22 cases of VUR detected on VCUG, 9 patients (41\%) had VUR grade I, 7 patients (32\%) Grade II, 2 patients (9\%) grade III, 2 patients (9\%) Grade IV and 2 patients (9\%) had grade V VUR. 
DISCUSSION: In our study totally 78 children were included. Among them $60 \%$ of the cases presented as recurrent UTI. Among total cases of UTI 34 (44\%) were males and 44 (56\%) were females, under 2 years the percentage of UTI in males and females were $53 \%$ and $47 \%$ respectively. The male to female ratio was 1.1:1.After that the ratio changed to 1:1.6 in between 3-5 years and 1:1.8 after 5 years of age. Thus with advancing age female children are at increased risk of getting UTI compared with male children. Similarly Hellerstein $\mathrm{S}^{9}$ also observed that during the first year of life the male to female ratio was 2.8-5.4:1 \& beyond 1-2 years there was a striking female preponderance with a male to female ratio of $1: 10$. Zamir $G$ et al ${ }^{10}$ also concluded in his study that the male to female ratio with first UTI, under 5 years of age was 1:3.

In children with first UTI most of them presented under 2 years (54\%) and $46 \%$ in the age group of 3-5 years. No cases of first UTI were seen after 5 years. A similar observation was made by Jack S. Elder ${ }^{12}$ who showed that first UTI usually occurs by the age of 5 years, with peaks during infancy and childhood.

Among clinical features under 2 years, fever was the predominant symptom with first UTI, followed by irritability, diarrhea and poor feeding as common presentations. In contrast with recurrent UTI fever was presented in only $28.5 \%$ cases. Irritability, vomiting, poor feeding and failure to thrive were other common presentations. After two years of age with recurrent UTI, increased frequency of urine $(77 \%)$ and dysuria were common presentations followed by abdominal pain and failure to thrive. In this study the proportion of VUR in UTI was $28.2 \%$. With Recurrent UTI32\% children were found to have VUR on VCUG. With first UTI the VUR was seen in only $22.5 \%$ cases. Zamir G et al ${ }^{10}$ also concluded in his study that $18.4 \%$ patients were found to have VUR on VCUG with first diagnosed, uncomplicated febrile UTI in all children under 5 years of age. Similarly the study conducted by Mahantet al ${ }^{11}$ also showed that $22 \%$ of children with UTI were found to have VUR on VCUG.

In our study the highest proportion of VUR were seen under 2 years of age, accounting for $35.2 \%$. As age increased the proportion decreased to $29 \%$ in 3-5 years of age and to $15 \%$ over 5 years. The results were similar to the study conducted by Deshpande PV et al. ${ }^{13}$ In his study $34 \%$ children under 1 year presented with VUR and $20 \%$ of children were found to have VUR on VCUG in between 1- 6 years of age. On analyzing the sex distribution of children with VUR, we found that among first UTI there is not much difference in distribution of VUR among males and females. With recurrent UTI, females presented with VUR more commonly than males especially under 2 years (Females $39.2 \%$, Males $20.5 \%$ ).

of 78 children who were included in our study, 8 children had mild to moderate renal pelvis dilation with ureteric dilatation on renal ultrasound. The results of our study showed that the sensitivity and specificity of sonography to diagnose the VUR were $36 \%$ and $100 \%$ respectively. Compared with the studies done by Mahantet al ${ }^{11}$ the sensitivity of USG correlated well with our studies. In his study the sensitivity and specificity of USG in detecting VUR were $40 \%$ and $76 \%$ respectively. Similarly Smellie and Rigden ${ }^{14}$ evaluated four methods of investigations in 58 children following UTI. 36 patients (62\%) were found to have VUR by VCUG, but only eight (13\%) had abnormal USG giving a sensitivity, specificity and false negative rate of $42 \%, 91 \%$ and $78 \%$ of renal ultrasound for predicting VUR.

In our study among 22 cases of VUR detected on VCUG 9patients (41\%) had VUR Grade I, 7 patients (32\%) Grade II, 2 patients (9\%) Grade III, 2 patients Grade IV and 2 patients (9\%) Grade V. Compared with the studies done by Zamir G et al the results fairly correlated with Grade I \& II. In his study Grade II VUR constituted 40\% cases, followed by Grade I and III which 
were $29 \%$ each. Grade IV VUR was found in only $2 \%$ of patients. Similarly compared with the study done by Kass ${ }^{15}$ and colleagues, only Grade II VUR correlated well with our studies. In his study 52\% VUR were Grade III, 35\% VUR Grade II, 9\% Grade I and No Grade V VUR was seen.

\section{REFERENCES:}

1. Chang SL, Shortliffe LD. Pediatric urinary tract infections. PediatrClin North Am. 2006; 53:379-400.

2. Smellie JM, Prescod NP, Shaw PJ, Risdon RA, Bryant TN. Childhood reflux and urinary infection: a follow-up of 10-41 years in 226 adults. PediatrNephrol. 1998; 12:727-36.

3. Indian Pediatric Nephrology Group. Revised statement on management of urinary tract infections. Indian Pediatr.2011; 48:709-17.

4. 4.Jakobsson B, Soderlundh S, Berg U. Diagnostic significance of 99mTcdimercaptosuccinicacid (DMSA) scintigraphy in urinary tract infection. Arch Dis Child 1992; 67(11):1338 - 42.

5. Cleper R, Krause I, Eisenstein B, et al. Prevalence of vesicoureteral reflux in neonatal urinarytract infection. ClinPediatr (Phila) 2004; 43(7):619 - 25.

6. Wennerstrom $\mathrm{M}$, Hansson $\mathrm{S}$, Jodal U, et al. Disappearance of vesicoureteral reflux in children. Arch PediatrAdolesc Med 1998; 152(9):879 - 83.

7. Jacobson SH, Hansson S, Jakobsson B. Vesico-ureteric reflux: occurrence and long-term risks.ActaPaediatrSuppl 1999; 88(431):22 -30.

8. Lebowitz RL, Olbing H, ParkkulainenKV,et al. International system ofradiographic grading of vesicoureteric reflux. International reflux study inchildren.PediatrRadiol 1985; 15:105-9

9. Hellerstein S. Urinary tract infections.PediatrClin North Am 1995; 42: 1433-1457.

10. Zamir G, Sakran W, Horowitz Y, Koren A, Miron D. Urinary tract infection is there a need for routine renal ultrasonography. Arch Dis child 2004;89: 466-468.

11. Mahant S, Friedman J, Mac Arthur C, Renal ultrasound findings and vesicoureteral reflux in children hospitalized with urinary tract infection. Arch Dis Child 2002; 86: 419 - 20.

12. Elder JS, Peters CA, Arant BS, Ewalt DH, Hawtrey CE, Hurwitz RS, et al. Pediatric vesicoureteric reflux guidelines panel summary report on the management of primary vesicoureteral reflux in children. J Uro 1997; 157: 1846 - 1851.

13. Deshpande PV, Verrier Jones K. An audit of RCP guidelines on DMSA scanning after urinary tract infection. Arch Dis child 2001; 84: 324-327.

14. Smellie JM, Rigden SPA, Prescod NP. Urinary tract infection: a comparison of four methods of investigation Arch Dis child 1995; 72: 247-50.

15. Kass EJ, Kernen KM, Caregy JM. Pediatric urinary tract infection and the necessity of complete urological imaging. BJU Int. 2000; 86: 94-6. 